\title{
American Society of Clinical Oncology 2021 Podcast: HR+, HER2- Breast Cancer
}

Kwok-Leung Cheung

\section{DIGITAL FEATURES}

This article is published with digital features, including a podcast video and audio file, to facilitate understanding of the article. To view digital features for this article go to: https://doi. org/10.6084/m9.figshare.15044019

\section{PODCAST TRANSCRIPT}

Victoria Glasson (VG): Managing Editor of Oncology and Therapy

Kwok-Leung Cheung (KLC): Professor of Breast Surgery and Medical Education, University of Nottingham

$V G$ : Hello and welcome to the Adis Podcast Series. Today, we're looking at the ASCO 2021 conference and the data released. We are talking to Professor Kwok-Leung Cheung of the University of Nottingham. Professor Cheung will be taking us through his highlights from the ASCO 2021 conference, specifically focusing on the data released on hormone receptor-positive, human epidermal receptor 2-negative breast cancer.

Supplementary Information The online version contains supplementary material available at https:// doi.org/10.1007/s40487-021-00164-2.

K.-L. Cheung $(\bowtie)$

University of Nottingham, Nottingham, UK

e-mail: Kwok_Leung.Cheung@nottingham.ac.uk
Thank you so much for joining us today, Professor Cheung. You are professor of breast surgery with expertise in primary breast cancer in older women at the University of Nottingham. Can you give us a quick overview of your role and your research interests?

$K L C$ : Well, thank you very much, Victoria. My role at the university is on breast cancer research, and also, I do a little bit of work on medical education. Clinically, I'm practicing as a breast surgeon in the UK. For my research, I have several themes for the program. But the main one is on, as you said, breast cancer in older women, which covers tumour biology according to different ages. Also, we look at the use of geriatric assessment tools to help optimizing the decision-making process for breast cancer. I also have involvement in clinical trials in endocrine and targeted therapies.

$V G$ : Brilliant. Today, you're going to be talking to us through your main highlights from the ASCO 2021 breast cancer data, looking at HR-positive, HER2-negative data from the conference. To get us started, what were your take-home messages from the early breast cancer data that was released at the conference?

$K L C$ : For the abstracts presented in ASCO on early breast cancer, the ER-positive and HER2negative ones, I think there are a couple of key points. One is on the use of genomics to aid deescalation of treatment. The other one is on the use of CDK4/6 inhibitors. Maybe we can start 
with the use of genomics to aid de-escalation of treatment. There were a couple of abstracts which were looking at the use of different genomic tools to see whether they could aid decision-making on extending adjuvant endocrine therapy beyond 5 years.

One was using the Breast Cancer Index, and the other one was using the 70-gene MammaPrint $[1,2]$. These tools were explored in the context of the NSABP B-42 trial. Unfortunately, the conclusions for both were a negative one, not showing any definitive roles by using these tools. I think genomics may help in this setting, but I don't think we are there yet at this point. Also, we may have to identify the precise assays to be used in different settings, meaning that, then, in different settings maybe there are different assays that we need to use.

$V G$ : There is an importance to being able to stratify beyond high or low risk, and to be able to see the genomic difference in tumour signatures, and really to start to understand them. Really, we're starting to look at taking a step closer to personalized treatment plans for patients and personalized therapy, which will be beneficial for patients if we're able to implement them. Would you agree? And the importance of having personalized medicinehow will that benefit patients in the future?

$K L C$ : I totally agree with you. The importance of this from the angle of tumour biology is that although breast cancer seems to be just one disease, effectively it isn't. There are differences in biology, for example, based on my research according to age. Also, there are different settings whereby the biology is different. So therefore, if you just apply your treatments based on a one-size-fits-all approach, even within ER-positive and HER2-negative disease, you are bound to either over-treat or under-treat a patient. Therefore, using these tools in order to identify the precise biology that would help you select treatment is very important. But unfortunately, we haven't got that as far as this conference is concerned.

$V G$ : Thank you. So next, you're going to talk us through the use of CDK4/6 inhibitor data in the adjuvant setting.

$K L C$ : Yes, indeed. As I will talk about this a bit later on the use of CDK4/6 inhibitors in metastatic disease, as you know, these compounds have been shown to be very, very useful when given alongside conventional endocrine therapies such as an aromatase inhibitor or the selective ER down-regulator fulvestrant in the metastatic disease setting. So therefore there has been a lot of interest in exploring their role in the adjuvant setting. There were some trials presented in ASCO this year looking at this subject, trying to use CDK4/6 inhibitors in the adjuvant setting alongside endocrine therapy.

There were two abstracts, they were all applying the concept in more high-risk settings in order to tease out whether the combination use of a CDK4/6 inhibitor with an endocrine therapy could actually produce any additional benefit. One was the setting whereby patients had high-risk disease, and so much so that they needed to receive neoadjuvant chemotherapy. Then, abemaciclib was used plus endocrine therapy. Interestingly, it did show a superior invasive disease-free survival. That on its own was very, very encouraging. That is based on the monarchE trial [3].

However, when the same concept was applied using palbociclib in premenopausal subgroup, patients in the PENELOPE-B trial, which, again, was seen to be probably a population with high-risk disease, no such benefits were seen in that study [4]. If you pulled the two together and considered the subject, so it looks as though individual CDK4/6 inhibitors are biochemically different, and we know that. Maybe they produce different clinical efficacies in different settings. We probably cannot say at this point in time that if one CDK4/6 inhibitor works in one setting it will work in all other settings.

Therefore, regardless of the individual agents, we have not yet seen any improvement in overall survival. For the first trial that I just mentioned, in monarchE [3], there was improvement in the invasive disease-free survival, which was the primary endpoint. However, there was not yet any evidence of improvement in overall survival, and given the fact that there was another CDK inhibitor which did not show the same thing, we are not quite ready in that sense. It's still premature to conclude that the adjuvant role of this 
compound, or class of compounds, is definitively confirmed at this stage.

$V G$ : Excellent, thank you. What were your main take-home points now from the metastatic breast cancer research?

$K L C$ : So for metastatic breast cancer, that's very, very interesting. I just finished talking about the adjuvant use of CDK4/6 inhibitors, whereby there was one trial showing an improvement with that combination with endocrine therapy when compared to endocrine therapy alone, but not consistently shown in another study. However, in metastatic disease for the same tumour type, ER-positive and HER2-negative disease, as we already know, all the existing three commercially available CDK4/6 inhibitors have been shown to have significant improvement in terms of progression-free survival when used in combination with an endocrine therapy when compared to using the endocrine therapy alone in metastatic disease setting.

But this year in ASCO, we got some important results from two trials which showed that there was, in fact, long-term overall survival benefits. Just to quote that, one trial was PALOMA-3, which was investigating the use of palbociclib alongside fulvestrant after prior endocrine therapy in the metastatic disease setting [5]. There was a significant improvement in long term overall survival benefit. The hazard ratio after a median follow-up of 73.3 months was 0.806 , and the 5 -year survival of the treatment arm-that means the combination armwas $23.3 \%$ as compared to $16.8 \%$ in the control arm, which was endocrine therapy alone.

The other trial, MONALEESA-3, which used ribociclib, another well-known CDK4/6 inhibitor, given alongside fulvestrant, this time as a first-line endocrine therapy, when compared to fulvestrant alone also showed similar results [6]. That's very encouraging in terms of long-term overall survival benefits. In this trial, what it showed was a median follow-up of 56.3 months, which was, again, very long term. So there was a significant benefit in terms of median overall survival benefits of 53.7 months versus 41.5 months, with a hazard ratio of 0.73 .

With these two studies in mind presented in ASCO, they are really important, because they were the first reports of long-term overall survival benefits. The superiority of this class of compounds plus endocrine therapy over endocrine therapy alone in this setting, which is metastatic disease, is now definitively confirmed, not only with the already known fulfilment of the primary endpoints, which was progression-free survival, but overall survival benefits have been shown beyond a number of years in these two cases, about 5 to 6 years across two large randomized controlled trials.

$V G$ : Visceral disease in patients with HRpositive HER2-negative advanced breast cancer can lead to concerns when it comes to endocrine therapy. But the overall survival data that we're seeing is starting to show benefits to patients. Would you agree?

KLC: That's correct. That was the most important take-home message or most important finding, I would say, in ASCO this year for this category of tumour types.

$V G$ : Excellent. Would you be able to take us through CDK4/6 inhibitors and new agents and the data that was discussed?

$K L C$ : Thank you. Yes, indeed. There was another trial, which was presented this year, called DAWNA-1, which investigated a new CDK4/6 inhibitor called dalpiciclib [7]. That was used alongside fulvestrant, in a similar concept to one of the trials that I mentioned before, after prior endocrine therapy, again, in the metastatic setting. What it showed was similar to what we had seen before, a superior progression-free survival when compared to fulvestrant alone. The side effect profile was very, very similar, and most side effects were manageable like the others, as in the other three CDK4/6 inhibitors that we saw before. Therefore, with that in mind, I suggest that this class of compounds, the CDK4/6 inhibitors, appears to show consistent benefits when used in combination with endocrine therapy as compared to endocrine therapy alone.

Now, but the interesting thing would be, or a clinician would ask, how can I choose between them? We have got three in the market, and we've got this one coming through in the future in the pipeline. There might be more. How can we decide? So if newer compounds under the same class are to be developed, I think further 
studies may have to be done to tease out individual differences in terms of both efficacy and safety, so clinicians know how to choose between them. Maybe some of them are more applicable in certain settings and some in others.

$V G$ : And the toxicity of CDK4/6 drugs has been a concern in the past. But would you say that that's due to the younger nature of the treatments, and with further experience of side effect management and discussing those side effects with patients, we'll start to see that improving?

$K L C$ : I would think so. I think there is no doubt that the combination of CDK4/6 inhibitor with endocrine therapy give you more side effects and toxicity when compared to endocrine therapy alone. Having said that, all these studies seem to suggest that the safety profile is acceptable and the side effects are generally manageable. So I think the patients have to be informed about this as a trade-off against an improved progression-free survival and, now, with an improved overall survival in a metastatic disease setting.

$V G$ : Yes. It's really discussing the importance of having those conversations with patients, I think, and allowing them to make an informed decision about the side effects that they may experience but the offset benefits that they may experience as well.

$K L C$ : Definitely, and I think if we go back to one of the points that you asked very early on about personalizing treatment and about the use of genomics, I think one of the potential things that we could do in the future might be using genomics or other similar tools to identify patients whose tumour would behave or would be controlled equally well with endocrine therapy alone. So we don't have to indiscriminately use a CDK4/6 inhibitor alongside an endocrine agent, which we know would have more side effects and toxicities.

$V G$ : Really, really interesting. And then, I think you're also going to discuss with us the use of CDK4/6 inhibitors in older women. So this is really a topic that's of a lot of interest to you.

KLC: Yes, indeed. Indeed. As I just said, it would be useful on one hand to find out whether there are situations whereby even in ER-positive HER2-negative disease whether we could just use endocrine therapy alone to achieve similar efficacy outcomes, so that we don't have to worry about the side effects. However, there might be situations whereby the combination is actually better or definitely better in certain situations. So therefore, the study called PALOMA is one of the studies looking at the use of palbociclib and endocrine agents.

There is an acronym called PALOMA-AGE or PALOMAGE, combining the term age with PALOMA, which actually looked at this subject in the older population [8]. It wasn't a randomized controlled trial, but it was using the concept of combining palbociclib with endocrine therapy in a real-world setting. They collected the data in the older population, even in those above the age of 80 . Some of them, though, had a lot of initial dose reductions, because it's real-world data; some clinicians made their own clinical judgment to reduce the dose when they started the treatment on those who were very old, like above the age of 80 .

What is showed here was that the study did show that the safety profile was reasonable and helped to alleviate concerns of using this class of compounds in the older population. But caution remains, especially for those very old or very frail. Some form of geriatric assessment should identify who these patients were, the very frail ones, so that intervention could be made in order for these treatments to be givenfor example, early dose reduction or initial dose reduction and/or closer monitoring during the course of treatments.

$V G$ : In the past we've seen discussion on how difficult it can be to recruit older women into clinical trials. This is something that you have written about yourself for the journal [9]. And older patients make up a small portion of the clinical trial recruitment. But the number of older patients living with cancer is increasing, and they're definitely underrepresented in clinical trials. This can have a knock-on effect onto the knowledge of the compounds that the older generation would be given. Is this something that you are starting to see change? Are 
there more older people being included in trial designs and research from the outset?

$K L C$ : I would say yes and no. Yes, in a sense that changes are happening because of the joint efforts of people like myself trying to push this like a vision. As you know, I have been involved in the work with the International Society of Geriatric Oncology. We've written a top priorities paper in Lancet Oncology this year [10], highlighting the importance of research in older adults with cancer. No, in the sense that the progress remains slow, and that's why we've got to keep trying and keep doing. Don't give up.

I think it's important to bear in mind that trial design could be difficult in the older population. So we need to bear in mind a few possible solutions or pragmatic solutions. Something like this one, this abstract, the realworld data is maybe one of the solutions [8]. Getting randomized controlled trials is one which would set the foundation to a concept or clinical concept. Then we've got to collect realworld data in a pragmatic way, especially in the older population, which could help steer things as well.

The other thing would be to continue recruiting patients in a randomized controlled trial setting, but make sure that there is no upper limit, for example, where we stratify patients according to age. Also, another thing which is important in the geriatric oncology world is to embed some sort of geriatric assessment into the trial design, so that we don't have to worry about someone who is frail, we better exclude them. Instead, we embed some sort of geriatric assessment, so that you can actually include them in a trial setting whereby you build in some sort of optimization strategiesfor example, in this case, dose reduction or close monitoring.

$V G$ : It's a really important point, I think. Would you agree that it needs the combined effort of the pharmaceutical companies and the researchers as well as patient advocacy groups to really try and inform decisions going forward into clinical trial designs?

$K L C$ : Indeed. Indeed. And in addition to all the stakeholders that you have just mentioned, I would just also like to mention that from the clinical side, collaboration between the oncology world and the geriatric world is also crucial.

$V G$ : Really, really interesting. Well, thank you so much, Professor Cheung, for that brilliant roundup of the data from the ASCO 2021. We thank you so much for joining us on the podcast today. We hope that that has been a really helpful roundup for all of our listeners. Please look out for other podcasts from the Adis Journal Podcasts.

\section{ACKNOWLEDGEMENTS}

Funding. This podcast has been developed through an unrestricted educational grant from Novartis. The author was selected by the journal, and the content of the podcast was developed independently by the author and the journal editor. The Rapid Service Fee was funded by an unrestricted educational grant from Novartis.

Authorship. The author meets the International Committee of Medical Journal Editors (ICMJE) criteria for authorship for this article, takes responsibility for the integrity of the work as a whole, and has given their approval for this version to be published.

Disclosures. Kwok-Leung Cheung is a member of Oncology and Therapy's editorial board but has nothing else to disclose.

Compliance with Ethics Guidelines. This article does not contain any studies with human participants or animals performed by any of the authors.

Peer Review. Please note, contrary to the journal's standard single-blind peer review process, as a podcast this article underwent review by a member of the journal's editorial board.

Open Access. This article is licensed under a Creative Commons Attribution-NonCommercial 4.0 International License, which permits any non-commercial use, sharing, adaptation, distribution and reproduction in any medium 
or format, as long as you give appropriate credit to the original author(s) and the source, provide a link to the Creative Commons licence, and indicate if changes were made. The images or other third party material in this article are included in the article's Creative Commons licence, unless indicated otherwise in a credit line to the material. If material is not included in the article's Creative Commons licence and your intended use is not permitted by statutory regulation or exceeds the permitted use, you will need to obtain permission directly from the copyright holder. To view a copy of this licence, visit http://creativecommons.org/licenses/by$\mathrm{nc} / 4.0 /$.

\section{REFERENCES}

1. Mamounas, E et al. Breast Cancer Index (BCI) and prediction of benefit from extended aromatase inhibitor (AI) therapy (tx) in $\mathrm{HR}+$ breast cancer: NRG oncology/NSABP B-42. https://meetings.asco. org/abstracts-presentations/201557. Accessed 17 June 2021.

2. Rastogi, P et al. Utility of the 70-gene MammaPrint assay for prediction of benefit from extended letrozole therapy (ELT) in the NRG Oncology/ NSABP B-42 trial. https://meetings.asco.org/ abstracts-presentations/201562. Accessed 17 June 2021.

3. Martin, $\mathrm{M}$ et al. Abemaciclib combined with adjuvant endocrine therapy in patients with high risk early breast cancer who received neoadjuvant chemotherapy (NAC). https://meetings.asco.org/ abstracts-presentations/197520. Accessed 17 June 2021.

4. Marmé, F et al. Palbociclib combined with endocrine treatment in breast cancer patients with high relapse risk after neoadjuvant chemotherapy: subgroup analyses of premenopausal patients in PENELOPE-B. https://meetings.asco.org/abstractspresentations/197603. Accessed 17 June 2021.

5. Cristofanilli, $M$ et al. Overall survival (OS) with palbociclib (PAL) + fulvestrant (FUL) in women with hormone receptor-positive $(\mathrm{HR}+)$, human epidermal growth factor receptor 2-negative (HER2-) advanced breast cancer (ABC): updated analyses from PALOMA-3. https://meetings.asco. org/abstracts-presentations/198379. Accessed 17 June 2021.

6. Slamon, D et al. Updated overall survival (OS) results from the phase III MONALEESA-3 trial of postmenopausal patients (pts) with HR+/HER2advanced breast cancer (ABC) treated with fulvestrant (FUL) \pm ribociclib (RIB). https://meetings. asco.org/abstracts-presentations/198455. Accessed 17 June 2021.

7. $\mathrm{Xu}, \mathrm{B}$ et al. Dalpiciclib versus placebo plus fulvestrant in HR+/HER2- advanced breast cancer that relapsed or progressed on previous endocrine therapy (DAWNA-1): a multicenter, randomized, phase 3 study. https://meetings.asco.org/abstractspresentations/195721. Accessed 17 June 2021.

8. Caillet, $\mathrm{P}$ et al. PALOMAGE, a French real-world cohort of elderly women beyond age 70 with advanced breast cancer receiving palbociclib: baseline characteristics and safety evaluation. https:// meetings.asco.org/abstracts-presentations/197621. Accessed 17 June 2021.

9. Cheung, KL et al. Current challenges faced by cancer clinical trials in addressing the problem of under-representation of older adults: a narrative review. https://link.springer.com/article/https:// doi.org/10.1007/s40487-021-00140-w. Accessed 17 June 2021.

10. Extermann, $M$ et al. Priorities for the global advancement of care for older adults with cancer: an update of the International Society of Geriatric Oncology Priorities Initiative. https://pubmed.ncbi. nlm.nih.gov/33387502/. Accessed 17 June 2021. 